\title{
Ratchet effect for cold atoms in an optical lattice
}

\author{
Emil Lundh and Mats Wallin \\ Department of Physics, Royal Institute of Technology, AlbaNova, SE-106 91 Stockholm, Sweden
}

(Dated: December 26, 2017)

\begin{abstract}
The realization of a directed current for a quantum particle in a flashing asymmetric potential is studied. It is found that a positive current, i. e. in the direction expected for a conventional diffusive ratchet, can be attained at short times in the limit where the potential is weak and quantum diffusion dominates, while current reversal is obtained for stronger potentials. A single parameter, the ratio between the kicking frequency and the optical lattice potential strength, determines both the degree to which quantum effects dominate, and the possibility of obtaining a ratchet current. The effect should be readily observable in experiments.
\end{abstract}

The study of nanoscale quantum pumps is of considerable current interest, motivated by both the quest to explore basic quantum phenomena, by recent significant experimental progress, and also by the potential practical implications in terms of artificial devices 11. Ratchet models have been introduced and studied for processes that allow extraction of work from diffusive motion without application of macroscopic gradients. For example, ratchets have been discussed in the context of biological motors [2] and for manipulating magnetic flux in artificially nanostructured superconductors 3 ].

With the advent of optical lattices, the possibility has opened up to study dynamics of systems of quantum particles in the absence of external noise. These provide a novel setting for ratchet effects and interest in purely quantum ratchets has therefore grown recently. In rocking ratchets, where an alternating bias field is imposed on the system, current reversal due to quantum effects has been observed [4, 5]. In an optical lattice, transport was observed in a setup that utilizes oscillations between internal states of the atoms to create an effective ratchet potential that alternates between two configurations [6]. Another realistic experimental setup for atoms in an optical lattice is a flashing ratchet, i.e., an asymmetric potential that is turned on and off periodically in time. Reference 7] carried out a theoretical study of such a system in the case of a small effective Planck constant, and found that no long-time transport was present in the case of strictly periodic flashing. They devised instead a scheme with unequal spacing between the flashes, which was shown to result in greatly enhanced transport. Reference [8] studied a similar system experimentally, but without any asymmetry in the potential, and found at short times a greatly enhanced spreading in momentum space due to quantum resonances at certain parameter values (however, because of the geometry, no directed transport could be attained).

In this paper we study a nonlinear quantum pump acting on a particle trapped in an optical lattice, driven by a flashing ratchet mechanism. The focus of the paper is to determine the conditions for directed transport in a one-dimensional flashing ratchet, in the regime where quantum effects are appreciable. The aim is to sort out the dependencies on the various parameters of the system and to provide physical explanations for the parameter dependences and the absence of long-time transport.

System - Consider a particle in one dimension subject to the Schrödinger equation [7, 8]

$$
i k \frac{\partial \psi}{\partial t}=-\frac{k^{2}}{2} \frac{\partial^{2} \psi}{\partial x^{2}}+K v(x) \sum_{n=0}^{\infty} \delta(t-n) \psi
$$

where

$$
v(x)=\sin x+\alpha \sin 2 x
$$

is the ratchet potential, which we assume to be composed of two standing laser waves. The parameter $\alpha$ controls the skewness of the periodic potential that is the origin of the ratchet effect. A value of $\alpha$ between 0 and 0.5 approximates a sawtooth potential where the sawteeth lean to the left; this is expected to yield a positive current in the usual case of classical diffusive motion. The profile is more asymmetric for larger values of $\alpha$. The potential is assumed to be flashed on and off at periodic intervals. To facilitate calculations we have taken the pulses to be delta functions in time. The case $\alpha=0$, corresponding to a pure sine potential, is formally equivalent to the kicked rotor and has been extensively studied theoretically and experimentally $8,9,10,11$. The addition of a second sine wave adds complexity to this classic problem and opens for the possibility of a ratchet effect [].

The units in Eq. (1) are chosen such that the temporal period of the flashing and the spatial period of the lattice are unity. In terms of physical quantities, the effective Planck constant is

$$
\hbar=8 \omega_{R} T,
$$

where $T$ is the period of the flashing and $\omega_{R}=\hbar k_{L}^{2} / 2 m$ is the recoil frequency of the applied laser field, with $k_{L}$ the recoil momentum giving a lattice period $\left(2 k_{L}\right)^{-1}$ for the optical potential felt by the atoms. The effective potential strength is

$$
K=k \frac{T E_{\mathrm{OL}}}{\hbar}=k P,
$$


where $E_{\mathrm{OL}}$ is the amplitude of the periodic potential of the laser beam with the lower frequency. We have implicitly defined the auxiliary quantity $P=K / \hbar$ in Eq. (4), because as we shall see, it determines much of the physics of the system. In addition, $P$ has a simple interpretation: it is just the ratio between the potential strength and the kicking frequency.

When the potential flashes are assumed to be deltafunction kicks, the time evolution can in principle be described analytically: in momentum space the wave function at time $t$ is given by

$$
\psi(k, t)=e^{-i \hbar k^{2} / 2} \sum_{q=-\infty}^{\infty} F(k-q) \psi(q, t-1),
$$

where the wavenumber difference $q-k$ is restricted to integer values because of the periodicity of the potential, and the function $F$ is given by []

$$
F(k)=\sum_{p=-\infty}^{\infty} J_{k-2 p}(P) J_{p}(\alpha P) .
$$

$J_{n}$ are Bessel functions. The dependency of $F$ on $P$ and $\alpha$ is suppressed for brevity. In the kicked-rotor case, $\alpha=0$, all terms except $p=0$ vanish in (6) and there results $F(k)=J_{k}(P)$. Although the analytical formula, Eqs. (56), is useful for studying analytical properties, it is easier to solve for the time evolution numerically using a FFT algorithm. We assume an initially homogeneous wave function with zero momentum. The time evolution will because of the periodicity bring in only momentum components $\psi(k)$ with integer $k$. The wave function is propagated in time, one period in each step, by first multiplying the wave function components in $k$ space by a phase factor $\exp \left(-i k k^{2} / 2\right)$, then Fourier transforming the wave function and applying the phase factor associated with the potential, $\exp (-i P v(x))$, to the real-space wavefunction before Fourier transforming back.

Effective Planck constant - Figure 1displays the mean wave number $\langle k\rangle$ of the wavepacket over the first $T_{\mathrm{av}}$ temporal periods, as a function of the effective Planck constant $k$. The magnitude of the $\sin 2 x$-term in the potential is chosen as $\alpha=0.3$. The main features of the wave number-vs-Planck constant curve do not depend critically on the value of $\alpha$, but as a rule the drift is stronger for larger $\alpha$. We have held $P$ constant, so that the potential strength in each of the panels of Fig. 1 varies along the abscissa as $K=P k$. Consider first the topmost panel, where $P=0.5$ and $T_{\mathrm{av}}=20$. The curve is seen to be dominated by a series of resonances, but overall there is positive transport in the anticipated direction. Shown is only the curve for $0<\hbar<4 \pi$, because it is periodic in $k$ with period $4 \pi$. When $P$ is kept constant, the potential-energy part of the time evolution operator does not depend on $\hbar$, but only the kinetic term $\exp \left(\hbar \hat{k}^{2} / 2\right)$ does, and since spatial periodicity restricts the values of
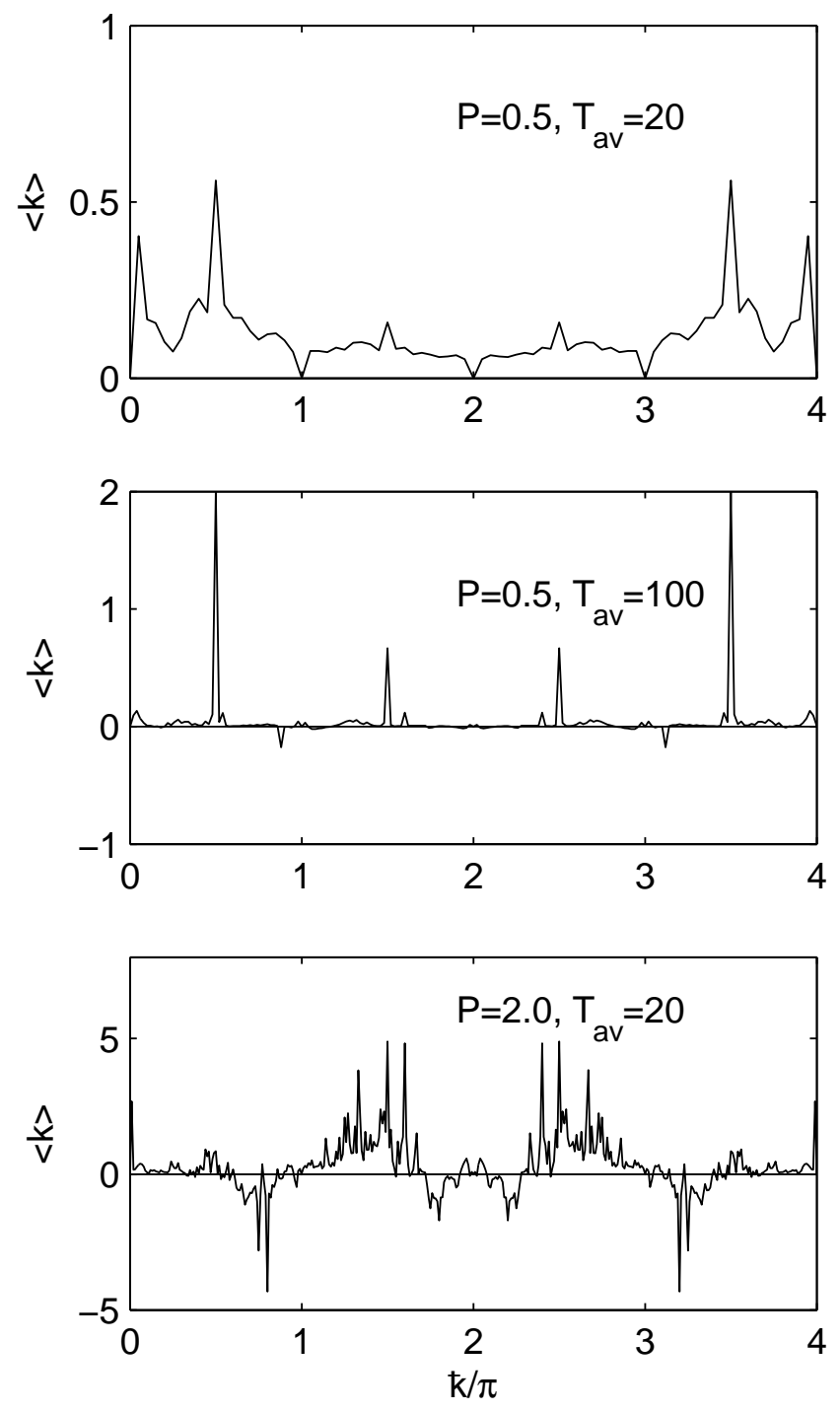

FIG. 1: Mean wave number of the wavepacket over the first $T_{\mathrm{av}}$ kicks, as a function of the effective Planck constant $k$. The asymmetry of the ratchet potential has been chosen to $\alpha=0.3$. Potential strength $P$ and averaging time $T_{\mathrm{av}}$ are as indicated in each panel.

$k$ to integers, the change $k \rightarrow \hbar+4 \pi$ does not affect the dynamics. It is thus not obvious that $k$ should be thought of as the parameter that determines whether the system is classical or quantum mechanical. Moreover, according to Eq. (5), changing $k$ to $-\hbar$ or $4 \pi-\hbar$ only results in an overall complex conjugation of the wave function and no change of the physics. This explains the reflection symmetry of the curve about the point $k=2 \pi$.

The resonances in $\hbar$ for integer and half-integer multiples of $\pi$ are well known from the kicked-rotor case, $\alpha=0$ [8, 9, 10]. The algebra pursued in Refs. 9, 10] can be carried over to the present, asymmetric case and yields similar results. Details of the calculation will be presented elsewhere [12]. 
Long-time behavior - The second panel in Fig. 1 shows the mean wave number over an averaging time of $T_{\mathrm{av}}=100$ kicks instead of 20 . Only the resonances remain in this curve; away from these the mean wave number has averaged out to nearly zero. Apparently, no transport can be expected at long times, except at resonances. This was also found in Ref. [7] and the reason is quantum localization. In the kicked rotor problem, it is well known that a quantum system will at short times undergo diffusion in momentum space, just like in the classical case, at a diffusion rate $D=K^{2} / 2$. However, after a so-called break time $t^{*} \propto D / k^{2}$, the quantum time evolution ceases to follow the corresponding classical evolution and the momentum distribution does not broaden any more. This is analogous to Anderson localization, although the phenomenon takes place in momentum space and not in real space [1]. The localization persists also in the case of an asymmetric potential, $\alpha \neq 0$, and seems to forbid a persistent current (see, however, Ref. [7]). Observe that with our definitions, the break time is simply $t^{*} \propto P^{2}$. In other words, a large $P$ means a more pronounced quantum-classical correspondence. The relevant parameter determining the quantum/classical character of the dynamics is thus $P$ and, perhaps somewhat counterintuitively, not $k$.

The present parameter value, $P=0.5$, is far into the quantum regime since it results in a break time $t^{*}$ less than one, and thus no quantum-classical correspondence can be expected. Figure 2 displays the time evolution of the wave packet for a representative choice of parameters away from resonances: $k=1.7$, and as before, $P=0.5$ and $\alpha=0.3$. The lower panel of Fig. 2 shows the momentum asymmetry, defined as the deviation of the density from its symmetrized value: $\delta n(k)=n(k)-(n(k)+n(-k)) / 2$. It is seen that the density distribution initially broadens, with a slight tendency towards positive $k$, whereafter the width of the distribution saturates due to localization, because of the short break time. From that point, the momentum distribution begins to oscillate back and forth within the wave packet, yielding a zero net momentum at longer times. The initial tendency towards positive $k$ is in accordance with our expectancies for a ratchet; this is further discussed below.

Kick strength - As discussed above, it is customary to discuss the physics of the present system in terms of the kick strength $K$, because the Schrödinger equation (11) assumes the intuitively expected form, and moreover, the effective Planck constant $k$ assumes the role of a quantum parameter. However, as we have seen, $P=K / \hbar$ is in fact the natural parameter that determines the degree to which the time evolution is quantum mechanical, and furthermore, as we now show, it turns out to determine whether a short-time ratchet effect is

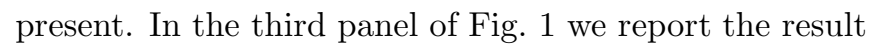
for slightly stronger kicks than in the examples above,
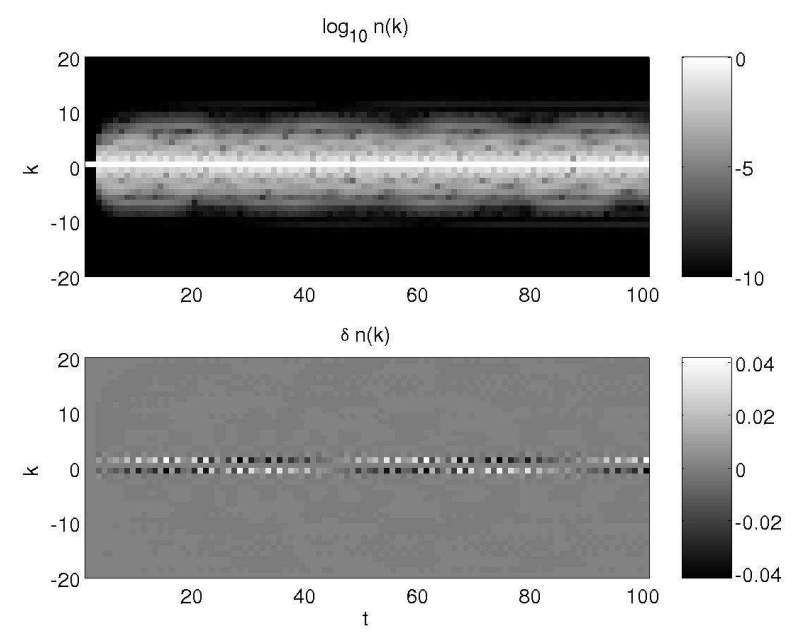

FIG. 2: Time evolution of the wave function in momentum space. Upper panel displays the density distribution; the logarithm is taken in order to enhance visibility. Each vertical cross-section of the plot represents the squared wave function $n(k, t)=|\psi(k, t)|^{2}$ at a time instant $t$. The lower panel shows the asymmetry in the momentum distribution, $\delta n(k, t)=n(k, t)-(n(k, t)+n(-k, t)) / 2$.

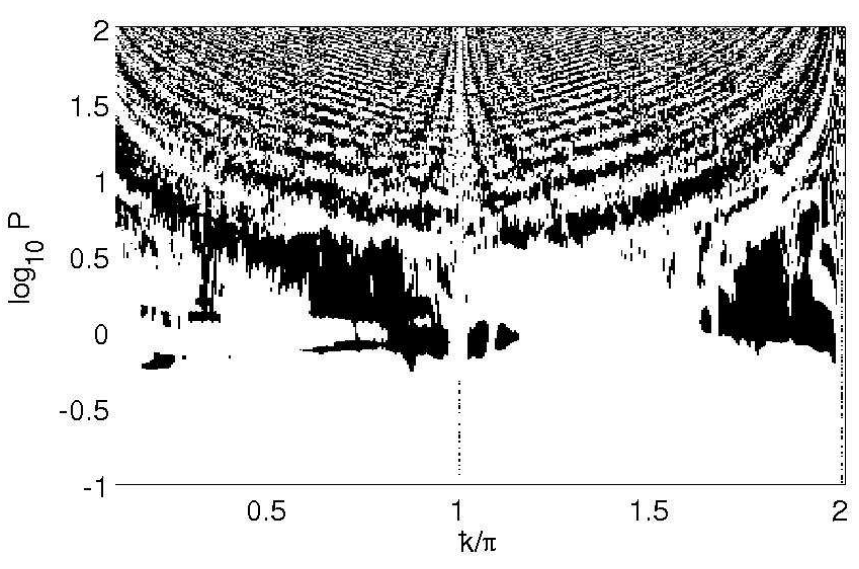

FIG. 3: Regimes of positive (white areas) and negative transport (black areas) for the quantum ratchet with $\alpha=0.3$. The mean momentum is taken over the first $T_{\mathrm{av}}=20$ periods. The first and third panels in Fig. 1] correspond to the horizontal cross-sections of the present curve at constant $P=0.5$ and $P=2$, respectively, while the middle panel of Fig. 1 is taken at a different $T_{\mathrm{av}}$.

$P=2$. The average is taken over twenty periods. For this kick strength, the curve has a considerably more broken appearance. In particular, the drift is no longer positive for all values of $k$ : it is just as likely to be negative. Indeed, we do find that for small values $P \lesssim 1$, the drift is always positive, except at anti-resonances, but it can be negative for larger $P$. Figure 3 shows the regions in $P-k$ space where the drift over the first twenty kicks is positive and negative, respectively. It is seen that for large values of $P$ or $K$ and for $0<k<\pi$, the black 
regions run along lines of constant $K$, indicating that in the classical regime, the dynamics depends on $K$ rather than $P$. On the other hand, what is interesting here is the dividing line below which the drift is purely positive, and that is clearly a line of constant $P$, not $K$.

The observation of negative transport (current reversal) for quantum ratchets in the zero-temperature limit has in the case of a rocked ratchet been explained with a tunneling argument 4]. That argument is not applicable for a delta-function flashing ratchet; obviously another mechanism is at play here. Consider the time evolution of a wavepacket originally concentrated within a well. Between two kicks, it undergoes diffusion according to the Schrödinger equation (11) and diffuses out to a final position uncertainty $\Delta x_{\text {diff }} \sim \hbar$ in the present units. The particle also receives an impulse from the periodic potential, which is of the order $\Delta p \sim K$ if the particle initially resided on one of the slopes of the potential, and hence travels a distance comparable to $\Delta x_{\text {ballistic }} \sim K$ in one period. We now see that the condition for a ratchet effect at short times,

$$
P \lesssim 1
$$

translates into a condition on the physical time scales,

$$
\Delta x_{\text {ballistic }} \lesssim \Delta x_{\text {diff }}
$$

In summary, we have investigated the conditions for directed transport for a quantum particle in a flashing ratchet potential. It is found that at short times, a directed current will flow in the anticipated direction if the kick strength $P$, defined as the ratio between the amplitude of the potential and the flashing frequency, is less than unity; this coincides with the limit in which the motion is inherently quantum mechanical, and quantum diffusion dominates over ballistic transport. It would be interesting to look for these findings in experiment: the levels of precision, noise and parameter control attainable in current experiments [6, 8 ] are certainly sufficient to test the predictions made in the present paper.

[1] P. Reimann, Phys. Rep. 361, 57 (2002).

[2] R. D. Astumian, Science 276, 917 (1997).

[3] J. F. Wambaugh, C. Reichhardt, C. J. Olson, F. Marchesoni, and F. Nori, Phys. Rev. Lett. 83, 5106 (1999); B. Y. Zhu, F. Marchesoni, and F. Nori, Phys. Rev. Lett. 92, 180602 (2004).

[4] P. Reimann, M. Grifoni, and P. Hänggi, Phys. Rev. Lett. 79, 10 (1997).

[5] H. Linke et al., Science 286, 2314 (1999).

[6] C. Mennerat-Robilliard et al., Phys. Rev. Lett. 82, 851 (1999).

[7] T. S. Monteiro, P. A. Dando, N. A. C. Hutchings, and M. R. Isherwood, Phys. Rev. Lett. 89, 194102 (2002).

[8] G. Duffy et al., e-print cond-mat/0401346 (2004).

[9] A. J. Daley and A. S. Parkins, Phys. Rev. E 66, 056210 (2002).

[10] F. M. Izrailev and D. L. Shepelyanskii, Theor. Math. Phys. 43, 553 (1980).

[11] F. Haake, Quantum Signatures of Chaos, 2nd ed. (Springer, Berlin, 2001).

[12] E. Lundh and M. Wallin, in preparation. 